\title{
O pescado na alimentação escolar, no Brasil: cenário, avanços e desafios
}

\author{
Fish in school meals in Brazil: scenario, advances and challenges \\ EI Pescado en la alimentación escolar en Brasil: escenario, avances y desafios
}

Recebido: 12/02/2021 | Revisado: 21/02/2021 | Aceito: 24/02/2021 | Publicado: 03/03/2021

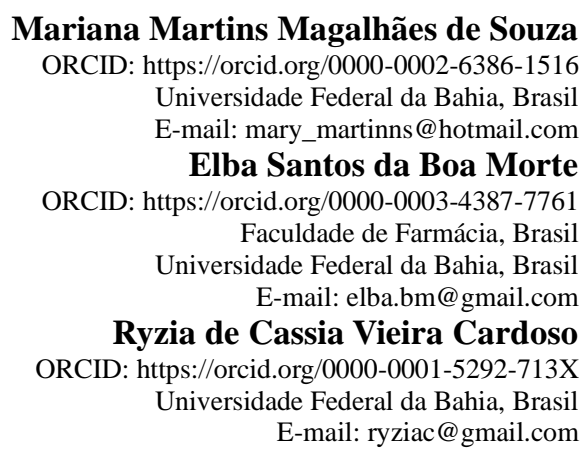

\begin{abstract}
Resumo
Este estudo objetivou abordar o cenário, os avanços e os desafios do consumo de pescado na alimentação escolar, no Brasil. Realizou-se uma revisão de literatura, abarcando diferentes tipos de publicações como artigos, livros, legislações e documentos de diversas organizações, considerando conteúdos estruturados em três eixos: o consumo de pescado no mundo e no Brasil; as diretrizes relacionadas ao uso do pescado na alimentação escolar; e o desenvolvimento de preparações à base pescado e testes de aceitabilidade, junto aos escolares, no Brasil. Conforme a literatura, no país, o consumo per capita/ano de pescado ainda é inferior à recomendação internacional (12 Kg per capita / ano). Políticas foram implementadas, buscando estimular este consumo pela população e na alimentação escolar, conquanto a coexistência desafios, na perspectiva cultural e operacional. Todavia, mudanças políticas e econômicas recentes têm repercutido negativamente nesta evolução. Nas escolas, a introdução do pescado mostrou-se constituir estratégia importante, por incentivar a formação do hábito do consumo deste alimento, desde a infância. Quanto aos estudos que desenvolveram e avaliaram a aceitabilidade de produtos à base de pescado, verificou-se que preparações como fishburguer, almôndegas e nuggets, ou mesmo bolos enriquecidos com filé de peixe foram bem aceitos, atendendo aos critérios para inserção nos cardápios das escolas. Apesar do cenário de retrocessos nas políticas de alimentação e nutrição do país, os estudos indicaram elevado potencial para a criação de preparações com pescado, com alta aceitabilidade pelos escolares.
\end{abstract}

Palavras-chave: Frutos do mar; Alimentação escolar; Hábitos alimentares; Criança.

\begin{abstract}
This study aimed at approaching the scenario, advances and challenges of seafood consumption in school feeding, in Brazil. A literature review was carried out, covering different types of publications such as articles, books, legislation and documents of several organizations, considering contents structured in three axes: seafood consumption in the world and in Brazil; guidelines related to the use of seafood in school feeding; and the development of seafood-based preparations and acceptability tests with schoolchildren, in Brazil. According to the literature, in the country, the consumption per capita / year of seafood is still lower than the international recommendation (12 Kg per capita / year). Policies have been implemented, seeking to stimulate seafood consumption in the country, while there are challenges from a cultural and operational perspective. However, recent political and economic changes have had a negative impact on this evolution. The introduction of seafood in schools showed to be important, given it encourages the formation of the eating habit to this food, since childhood. As for the studies which developed and evaluated the acceptability of seafood products, it was found that preparations such as fishburger, meatballs and nuggets, or even cakes enriched with fish fillets were well accepted, fitting criteria for insertion in school's menus. Despite the scenario of setbacks in the country's food and nutrition policies, studies have indicated a high potential for creating seafood preparations, with high acceptability by schoolchildren.
\end{abstract}

Keywords: Seafood; School feeding; Eating habits; Children.

\section{Resumen}

Este estudio tuvo como objetivo abordar el escenario, los avances y los desafíos del consumo de pescado en la alimentación escolar, en Brasil. Se realizó una revisión de la literatura, cubriendo diferentes tipos de publicaciones 
como artículos, libros, legislación y documentos de diferentes organismos, considerando contenidos estructurados en tres ejes: el consumo de pescado en el mundo y en Brasil; directrices relacionadas con el uso de pescado en la alimentación escolar; y el desarrollo de preparaciones a base de pescado y pruebas de aceptabilidad con niños en Brasil. Según la literatura, en el país, el consumo per cápita / año de pescado sigue siendo inferior a la recomendación internacional (12 Kg per cápita / año). Se implementaron políticas, buscando estimular el consumo en la población y en la alimentación escolar, a pesar de la convivencia de desafíos, en la perspectiva cultural y operativa. Sin embargo, los cambios políticos y económicos recientes han tenido un impacto negativo en esta evolución. La introducción del pescado en las escuelas resultó ser una estrategia importante, ya que favorece la formación del hábito de consumir este alimento, desde la niñez. En cuanto a los estudios que desarrollaron y evaluaron la aceptabilidad de productos a base de pescado, se encontró que preparaciones como hamburguesa de pescado, albóndigas y nuggets, o incluso tortas enriquecidas con filete de pescado fueron bien aceptadas, cumpliendo con los criterios de inclusión en los menús escolares. A pesar del escenario de retrocesos en las políticas de alimentación y nutrición del país, los estudios han señalado un alto potencial para la creación de preparaciones con pescado, con alta aceptación por parte de los niños en las escuelas.

Palabras clave: Mariscos; Alimentación escolar; Hábitos alimenticios; Niños.

\section{Introdução}

O consumo anual per capita de pescado tem aumentado ao longo do tempo, em nível mundial, contribuindo para a melhoria da qualidade da alimentação, posto que compreende espécies diversas, com valor nutritivo bem reconhecido (Food and Agriculture Organization [FAO], 2016). O pescado é considerado um alimento fonte de proteínas, além de oferecer outros nutrientes fundamentais para saúde humana, tais como vitaminas, minerais e ácidos graxos essenciais. Os peixes, em especial, são ricos em ácidos graxos essenciais - ômega-3 e ômega 6, sendo recomendado como fonte de gordura saudável (Domingo, 2007; Araújo, Montebello, Botelho \& Borgo, 2008; Jabeen \& Chaudhry, 2011).

Nos países em desenvolvimento, o consumo de pescado ainda é considerado inferior àquele dos países desenvolvidos. Nos primeiros, esse consumo depende dos produtos que estão disponíveis nos locais, sendo impulsionado mais pela oferta do que pela procura e associado ao aumento da renda nacional. Em adição, estes países têm experimentado um aumento da disponibilidade de pescado, por meio do crescimento das importações (FAO, 2016).

No Brasil, apesar da contribuição nutricional do pescado, o consumo ainda está abaixo do recomendado pela Organização Mundial de Saúde (OMS) - $12 \mathrm{Kg}$ per capita/ano, registando-se média inferior a 10Kg de pescado per capita por ano (Peixe BR, 2019).

No país, as crianças representam a faixa etária com menor consumo de pescado. Assim, torna-se importante o desenvolvimento de estratégias de intervenção, em educação alimentar e nutricional, voltadas para a melhoria da qualidade da alimentação, a partir da inserção de pescado, no âmbito familiar e no escolar (Godoy et al., 2010). Nesse contexto, cabe considerar que, além da família, a escola é um ambiente propício para estimular o consumo de alimentos saudáveis, que podem não ter sido oferecidos anteriormente à criança pela família. Um desses alimentos é o pescado, que também não se faz muito presente nos cardápios escolares do país, tornando-se necessário estimular o seu consumo pelos alunos (Sartori \& Amancio, 2012).

Em estudo de Mazzutti e Ferreto (2006), em Salto do Lontra-PR, foi observado um baixo consumo de peixe entre as crianças menores de 24 meses, verificando-se que 50\% destas não faziam o consumo e que $25 \%$ ingeriam apenas uma vez na semana. No estudo Hinnig e Bergamaschi (2012), igualmente, foi constatada a baixa frequência do consumo de peixes, importantes fontes de proteínas, entre escolares de 7 a 10 anos, em uma escola pública de São Paulo-SP, contribuindo com menos de $2 \%$ do consumo deste macronutriente.

Dado o contexto, o Fundo Nacional de Desenvolvimento da Educação (FNDE) (2013), executor do Programa Nacional de Alimentação Escolar (PNAE), e o Ministério da Pesca e Aquicultura firmaram, em 2013, um acordo para promover a inclusão do pescado na alimentação escolar. Esse acordo estabelecia diversas ações, como a capacitação de atores 
envolvidos no processo, incluindo manipuladores de alimentos, gestores, nutricionistas, professores e fornecedores/produtores de pescado provenientes da pesca artesanal e da aquicultura familiar, além da elaboração de cartilhas e materiais educativos que incentivavam o consumo do pescado entre crianças e jovens.

Segundo a FAO (2016), a inclusão do pescado na alimentação de crianças pode auxiliar no combate de doenças crônicas degenerativas, além de modificar os hábitos alimentares e promover uma alimentação mais saudável. Esta diretriz encontra-se referendada politicamente em vários países, sobretudo considerando particularidades da composição do pescado e a sua essencialidade para promoção da saúde humana (Wine, Osornio-Vargas \& Buka, 2012; Food and Drug Administration [FDA], 2017).

Nos países em desenvolvimento da América Latina e Caribe, ainda, a inserção do pescado local na alimentação escolar constitui também uma estratégia que contribui para a melhoria da renda das famílias de pescadores artesanais e aquicultores, que têm recursos limitados. Desta forma, o estímulo ao consumo desdobra-se como apoio aos pequenos pescadores artesanais, que desempenham papel chave na pesca sustentável e na segurança alimentar (FAO, 2016).

Mediante o contexto explicitado, este estudo tem por objetivo avaliar o cenário, os avanços e os desafios do consumo de pescado na alimentação escolar, no Brasil.

\section{Metodologia}

Foi realizada uma revisão de literatura, abarcando diferentes tipos de publicações -artigos científicos, livros e legislações - bem como documentos de diversas organizações que abordassem os temas a seguir: i. o consumo de pescado no mundo e no Brasil; ii. as diretrizes relacionadas à alimentação escolar voltadas ao uso do pescado; e iii. o desenvolvimento de preparações à base pescado e testes de aceitabilidade, junto aos escolares, no Brasil.

Quanto ao recorte temporal, para os temas i e ii., realizou-se busca livre. Para os temas iii e iv, as buscas delimitaram os anos de 2000 a 2020, pois compreende o período a partir do qual se nota uma maior preocupação com o consumo e o aproveitamento do pescado, no país.

As bases de dados para a pesquisa incluíram: Scientific Electronic Library Online - SciELO, Medical Literature Analysis and Retrieval System Online (MEDLINE) e Scopus. Para a busca, utilizou-se a combinação dos seguintes termos, em português, inglês e espanhol: frutos do mar, peixe, pescado, valor nutricional, saúde, consumo, política, merenda escolar, alimentação escolar, desenvolvimento de novos produtos, produtos de pescado, avaliação sensorial. Também foram selecionados artigos, a partir de estudos identificados.

Em relação aos produtos elaborados à base de pescado, foram incluídos apenas estudos que testaram a aceitabilidade apenas com crianças e adolescentes. Assim, foram excluídos estudos que elaboraram preparações com pescado, mas que não realizaram o teste de aceitabilidade e aqueles cujo teste foi realizado apenas com adultos. Além disso, excluíram-se estudos que avaliaram os benefícios do consumo de pescado para patologias específicas, aqueles que reportavam dados de produção de pescado e os que tratavam de alimentação escolar, sem abordar o pescado.

Neste caso, ainda, os estudos selecionados foram analisados considerando os seguintes aspectos: autor(es); ano de publicação; local do estudo; público-alvo; método de avaliação da aceitabilidade; produtos desenvolvidos; método de cocção; e resultados obtidos.

\section{Resultados e Discussão}

\subsection{Consumo de pescado no mundo e no Brasil}

O pescado que compreende os peixes, moluscos, crustáceos e outros grupos de água doce ou salgada destinados a 
alimentação humana constituindo uma fonte alimentar rica em proteínas de alto valor biológico, de fácil digestibilidade e possui uma variedade de vitaminas, minerais e ácidos graxos essenciais como o ômega 3 (Tacon \& Metian, 2013).

Na perspectiva da saúde humana, os ácidos graxos da família ômega 3 são componentes essenciais na prevenção de doenças cardiovasculares, pois reduzem a suscetibilidade do coração à arritmia ventricular, reduzem a pressão arterial e têm efeito antitrombogênico e hipotriglicerídico (Kris-Etherton, Harris \& Appel, 2002; Sinn, Milte \& Howe, 2009; Raatz \& Bibus, 2016). Ainda, desempenham papel importante na modulação de processos inflamatórios e atuam diretamente na fluidez da membrana neuronal e na função do receptor, sendo a sua deficiência associada com várias doenças neurológicas e psiquiátricas, incluindo: a doença de Parkinson, a depressão, o transtorno bipolar, a demência, a Doença de Alzheimer, a esquizofrenia, o transtorno de déficit de atenção/ hiperatividade e o autismo (Lange, 2020).

Os benefícios do consumo regular de pescado têm sido cada vez mais reconhecidos, no entanto, alguns fatores limitam o seu consumo. No entanto, alguns fatores ainda limitam o consumo de pescado no Brasil (Verbeke, Vermeir \& Brunsø, 2007).

O consumo per capita mundial de pescado tem apresentado aumento, ao longo das últimas décadas, sendo registrado 9,0 kg, em 1961, e 20,3 kg, em 2017, com uma taxa média de crescimento de aproximadamente 1,5\% ao ano (FAO, 2020). Alguns fatores estão relacionados a essa elevação do consumo, como: o aumento da produção; avanços tecnológicos na fabricação, cadeia de frio, transporte e distribuição; o aumento da renda em todo o mundo, que está intimamente associada ao aumento da procura por pescado e produtos da pesca; redução de perdas e desperdícios; e a maior conscientização sobre os benefícios para a saúde do pescado, entre os consumidores (FAO, 2020).

Em países asiáticos, como Bangladesh, China, Índia, Indonésia, Filipinas, Tailândia e Vietnã, da ingestão total de proteína animal, entre $15 \%$ e $53 \%$ são provenientes de pescado, sendo que a aquisição é variável, tanto em termos de consumo per capita quanto de espécies de pescado, em função da renda dos domicílios. Assim, os grupos com renda mais baixa ingerem mais pescado de menor custo, enquanto as pessoas com maior poder aquisitivo gastam mais com espécies de pescado mais caras. Vale destacar que esse consumo é consideravelmente maior nas áreas rurais do que nas áreas urbanas (Mohan Dey et al., 2005).

No estudo de Welch et al. (2002), realizado em 10 países europeus, verificou-se maior utilização de peixe branco na Espanha e na Grécia e de peixe gordo nas zonas costeiras do norte da Europa (Dinamarca, Suécia e Noruega) e na Alemanha. Além disso, a maior ingestão de crustáceos foi encontrada nos centros franceses, espanhóis e italianos. Os autores reportam uma variação geográfica significativa na ingestão total e nos subgrupos e espécies de pescado consumidas em toda Europa.

Nos países em desenvolvimento, nos quais a pesca artesanal é marcante, a aquisição de pescado atrela-se fortemente à oferta de produtos locais. Por outro lado, em economias emergentes, devido ao aumento na renda nacional, os consumidores têm experimentado diversificar os tipos de pescado disponíveis, a partir de importações (FAO, 2016).

No Brasil, levantamentos têm revelado maior consumo de pescado pela população. Em 2001, o valor médio consumido foi de 6,79 $\mathrm{Kg}$ por habitante (Brasil, 2014a). Em 2019, registrou-se média inferior a 10Kg de pescado per capita, estando abaixo da recomendação da OMS (Peixe BR, 2019). Vale ressaltar, ainda, que o consumo difere entre as regiões do país (Sociedade Nacional de Agricultura, 2015).

Para Bombardelli, Syperreck e Sanches (2005) e Galvão e Oetterer (2014), fatores como o alto custo, os tabus alimentares, a dificuldade de preparo e a baixa qualidade de conservação do pescado, limitam o seu consumo no Brasil.

Apesar de não ter alcançado a recomendação da OMS, o aumento do consumo no Brasil pode ser atribuído, em parte, ao aumento da renda do brasileiro, bem como um conjunto de ações de incentivo, promovidas pelo extinto Ministério da Pesca e Aquicultura (MPA), no período anterior à recessão econômica de 2015-2018 (Brasil, 2014a).

Dentre estas iniciativas, constaram: o Programa Caminhão do Peixe, que disponibiliza veículos dotados de câmaras 
frigoríficas para comercialização de pescado congelado e fresco, a Feira do Peixe, que conta com módulos metálicos desmontáveis e utensílios, e o Kit Feira para comercialização do pescado em feiras e mercados públicos, que possibilitam a venda direta do pescado, sem intermediários (Silva, 2012).

Outra iniciativa, ainda, é a Semana do Pescado, evento anual, que busca incentivar o comércio de pescado com preços mais baixos e a distribuição de material educativo, com informações sobre compra, manipulação, conservação e valor nutricional do pescado. Em paralelo, há uma ação de fomento à inserção de itens da pesca artesanal na alimentação escolar, incentivando as crianças a consumirem produtos saudáveis, incluindo o pescado (Silva, 2012; Semana do pescado, 2020).

Cabe pontuar, que, no Brasil, o setor pesqueiro industrial não é bem distribuído. Conforme levantamentos, nas regiões Norte e Nordeste, a pesca artesanal é marcante, enquanto quase toda produção industrial é destinada à exportação. De modo inverso, verifica-se cadeia industrial mais concentrada nas regiões Sudeste e Sul, voltada ao beneficiamento do pescado, incluindo tecnologias de resfriamento, congelamento, salga, enlatamento e elaboração de subprodutos (farinha e óleo) para o mercado interno (Brasil, 2014a; Silva, Vieira, Simões \& Abdallah, 2005).

Nesse contexto, Amaral, Aparício, Souza e Santos (2017) reforçam a necessidade de ampliar o emprego de tecnologias de processamento, ofertando aos consumidores produtos mais elaborados e apresentáveis, que sejam de rápido e fácil preparo, como hambúrgueres, nuggets, patês, salsichas e demais produtos semiprontos, como uma das formas de estimular o consumo de pescado.

\subsection{O pescado e as políticas públicas voltadas para a alimentação escolar no Brasil}

Apesar de constituir importante fonte de proteína animal, em todo o mundo, com ingestão associada a uma menor chance de doenças crônicas, há indicações do pescado não ser apreciado pelos escolares ou valorizado pelos familiares responsáveis. Em geral, os familiares e os cuidadores apresentam grande restrição em fornecer peixe às crianças, pelo receio de acidentes com a ingestão de espinhas (Veit et al., 2012).

Guimarães, Quaresma, Sonati, Zica e Maciel (2015), ao avaliarem o consumo de pescado e sua associação com a composição corporal, nível de atividade física e percepção da qualidade de vida, em escolares de uma escola pública na cidade de Palmas-TO, constataram que a maioria (41,93\%) dos escolares consumiam pescado uma vez na semana e que $12,9 \%$ nunca consumiam pescado. As principais razões apontadas para o baixo consumo foram o preço elevado e a falta de hábito, podendo um ser uma consequência do outro.

No Brasil, tendo em vista que as crianças compreendem o grupo que apresenta o menor consumo de pescado, salientase a necessidade de inserir este tipo produto na alimentação escolar, como uma das estratégias para melhorar a qualidade da dieta das crianças, bem como associar o trabalho de educação nutricional para estimular o consumo (Godoy et al., 2010). Nesse contexto, ressalta-se a escola como um ambiente propício para a construção e a consolidação de práticas alimentares saudáveis, uma vez que as atividades direcionadas à educação em saúde podem gerar grande repercussão (Pérez-Rodrigo \& Aranceta, 2001).

De acordo com a Lei no 11.947/2009 e a Resolução CD/FNDE nº 26/2013, que regulamentam o PNAE, pelo menos $30 \%$ dos recursos do FNDE devem ser usados para a aquisição de alimentos diretamente da agricultura familiar. Estas normas são consideradas um marco para o apoio ao desenvolvimento sustentável local, pois, além de incentivar a compra de diversos alimentos produzidos localmente, estimula hábitos alimentares regionais e saudáveis como é o caso do consumo do pescado (Amorim, Rosso \& Bandoni, 2016).

Além disto, houve uma parceria entre MPA e o Ministério do Desenvolvimento Social e Combate à Fome (MDS), a fim de aumentar as compras de pescado, por meio do Programa de Aquisição de Alimentos (PAA), do Governo Federal. Como objetivo de longo prazo, buscou-se a inserção do pescado no Programa Nacional de Alimentação Escolar, com o propósito de 
estabelecer uma nova cultura alimentar (FNDE, 2013).

De modo alinhado a esta política Lima, Pinto, Kato e Pires (2017) realizaram estudo em uma escola de ensino fundamental, em Palmas-TO, a fim de valorizar o pescador e incentivar o consumo de peixe, estimulando o hábito saudável entre escolares. No trabalho, foi realizada peça teatral e paródia, abordando informações nutricionais referentes ao pescado e a relevância de seu consumo, assim como seus benefícios em nível de visão, ossos e capacidade cognitiva, sendo as informações demonstradas de forma direta e lúdica. Os autores reportaram êxito no estudo, constatando-se um aumento significativo de respostas corretas sobre os benefícios de consumo do pescado pelos escolares, após a intervenção, estimulando, deste modo, a introdução de pescado na alimentação.

Neste contexto, salienta-se a importância das ações de educação alimentar e nutricional que envolvam, além das crianças, os pais, posto que são consideradas mais eficazes. Dentre as metodologias, podem ser utilizadas: a entrega de folhetos sobre composição nutricional, o incentivo à alimentação saudável nos domicílios, carta com orientação para motivação e o encorajamento das crianças a comerem opções saudáveis e outras atividades interativas e divertidas, como histórias educativas, desenhos, jogos, práticas culinárias e de degustação (Nekitsing, Hetherington \& Blundell-Birtill, 2018).

Em pesquisa feita pelo Ministério da Pesca e Aquicultura (MPA), junto ao FNDE, referente à inclusão do pescado na alimentação escolar, em 2011, observou-se que, de 2000 municípios, apenas 34\% registraram o pescado na alimentação escolar. Entre as dificuldades encontradas para a inclusão estavam a baixa aceitabilidade/falta de hábitos pelos alunos e o preço elevado (36\% para cada), seguidas do risco de espinhas (28\%), dificuldade de acesso (17\%) e ausência de fornecedores e de produtos $(15 \%)$. Ainda, foi constatado que os peixes inteiros e eviscerados, assim como os moluscos e crustáceos, eram pouco frequentes, sendo a frequência mensal de consumo a mais relatada (42\%), seguida pela quinzenal (31\%) (Nota técnica n.004, 2013).

Conforme a Agência Brasil (2012), ainda, diversas dificuldades têm sido apontadas para baixa inclusão do pescado nos cardápios da alimentação escolar, como a ausência/insuficiência de câmaras frigoríficas nas escolas e a falta de habilidade das merendeiras na preparação do pescado. Soares (2015) e Melão (2012) referem algumas barreiras que são encontradas desde as etapas de aquisição, como a elaboração de chamada pública e do projeto de venda e conformidade com os padrões de qualidade, bem como no que tange aos investimentos dos gestores locais, à compreensão da importância da inclusão de alimentos agroecológicos na alimentação escolar, à adaptação dos agricultores familiares, ao aperfeiçoamento na logística de entrega e à regulamentação dos alimentos ofertados.

Dificuldades para inserção de produtos de origem marinha em programas de alimentação escolar também foram identificados nos Estados Unidos, por Virta e Love (2020), que destacaram como desafios o financiamento sustentável do programa, a rede de distribuição de frutos do mar, o desenvolvimento de receitas e o maior custo do pescado, em comparação com outras proteínas. Nessa direção, os estudiosos consideraram dificuldades para inserir e manter os programas, demandando auxílio de diversos setores, de modo a permitir tanto a incorporação do pescado nos cardápios escolares quanto o desenvolvimento de produtos pré--preparados para alimentação escolar.

Esses achados são reforçados por O'Hara e McClenachan (2019), ao pontuar que as compras de pescado pelas escolas americanas são raras e que o conhecimento sobre as barreiras para a sua aquisição local pode indicar estratégias de apoio a estas práticas. Segundo os autores, a compra de pescado por escolas próxima aos portos pesqueiros, pode constituir estratégia importante para melhorar a sustentabilidade do consumo, promovendo a formação de hábitos alimentares saudáveis e duradouros e a educação interativa sobre os sistemas de produção das espécies marinhas.

No Brasil, além das dificuldades elencadas, cabe considerar, em uma perspectiva mais recente, mudanças na conjuntura política e econômica do país, a partir de 2016, que resultaram em um continuum de fragilização das políticas públicas de alimentação e nutrição. Este quadro persistiu e foi acentuado no governo subsequente, sendo caracterizado por 
processos de quebra institucional e de programas, cortes financeiros e retrocessos em direitos adquiridos. Esses cortes abrangeram os programas e metas do Plano Nacional de Segurança Alimentar e Nutricional, incluindo o Programa de Aquisição de Alimentos (PAA), cuja redução orçamentária entre os anos de 2014 e 2018 foi de 67\% (Vasconcelos et al., 2019). Nesse sentido, o país passou a apresentar retrocessos na condução de políticas relativas ao PAA e ao PNAE.

Em estudo mais recente, Sambuichi, Almeida, Perin, Moura e Alves (2020) reportaram drástica redução dos recursos destinados ao PAA, registrando, para 2018, cerca de um quinto do valor aplicado em 2012. Para 2019, os autores consideraram haver valores ainda menores que os de 2018, conquanto as estimativas ainda não tivessem concluídas para o período.

\subsection{Elaboração e aceitação de preparações à base pescado na alimentação escolar, no Brasil}

No período entre 2011 e 2020 foram identificados onze estudos que reportam o desenvolvimento de produtos à base de pescado e a aplicação de teste de aceitação com alunos, com vistas à inserção das preparações na alimentação escolar, não sendo identificados estudos nos anos de 2014 e 2019 (Tabela 1).

Dentre os trabalhos verifica-se que a maioria $(45,45 \%)$ foi realizado na região Sul, onde se descreve costa mais favorável à pesca e maior disponibilidade de tecnologias pesqueiras (Silva, Vieira, Simões \& Abdallah, 2005; Perez et al., 2002). No Norte e no Nordeste, por outro lado, registra-se uma larga produção de pescado da pesca artesanal e menor produção industrial (mais voltada à exportação), o que pode constituir limitações para o uso do pescado na alimentação dos escolares.

Entre os produtos testados, constatou-se a utilização de peixes sob a forma de farinhas, sopas, produtos cominutados e reestruturados - almôndegas, fishburguer, nuggets, e, ainda, o peixe empanado. Seguindo a tradição cultural, as preparações com pescado incluíram sobretudo, formulações destinadas ao almoço, ou como proteína principal ou acompanhando massas. O uso em preparações doces (bolo e biscoito) foi menor (Tabela 1).

Boscolo, Feiden, Maluf e Veit (2009) elencaram diversas receitas que podem ser elaboradas a partir de peixes, com vistas à alimentação escolar, especialmente aqueles provenientes da água doce, como pacu e tilápia, envolvendo tanto preparações típicas de almoço, como macarrão, lasanha, escondidinho, farofa e outras, quanto algumas na forma de lanches (quibe, bolo, patê e outras).

Com relação ao uso da carne de peixe triturada ou mecanicamente separada, como no caso das almôndegas, do fishburguer e dos nuggets, destaca-se a disponibilidade de uma fonte proteica livre de escamas e espinhas, o que reduz significativamente os riscos de acidentes associados ao consumo de peixes. Em adição, é fato a tendência de aumento no consumo de hambúrgueres, empanados e diversos pratos pré-preparados e semi-prontos, no Brasil (Komiyama et al., 2009).

Neste caso, o uso de peixes de baixo valor comercial também é uma opção, permitindo o desenvolvimento de derivados de peixe, como hambúrguer e nuggets, que ao acrescentar ingredientes e especiarias resultam em um sabor agradável e boas características sensoriais e nutricionais (Ribeiro, Oliveira, Ferreira, Pereira \& de Silva, 2009).

Mediante o conjunto de preparações apresentadas nos estudos e a variedade de classes que compõem o grupo do pescado, entretanto, verifica-se que todos os produtos foram desenvolvidos empregando apenas os peixes, o que ainda permite explorar o potencial do uso de outros tipos de pescado, como moluscos e crustáceos, para inserção na alimentação das escolas. 
Tabela 1. Produtos desenvolvidos à base de pescado dos estudos para inserção na alimentação escolar. Brasil, 2021.

\begin{tabular}{|c|c|c|c|c|c|c|}
\hline Estudo & Local & $\begin{array}{l}\text { Público- } \\
\text { Alvo }\end{array}$ & $\begin{array}{c}\text { Avaliação da } \\
\text { Aceitabilidade }\end{array}$ & $\begin{array}{c}\text { Produto } \\
\text { Desenvolvido }\end{array}$ & $\begin{array}{l}\text { Método de } \\
\text { Cocção }\end{array}$ & Principais Resultados \\
\hline $\begin{array}{c}\text { Borges, } \\
\text { Passos, } \\
\text { Stedefeldt e } \\
\text { Rosso (2011) }\end{array}$ & Santos, SP & $\begin{array}{c}93 \text { alunos } \\
\text { do } \\
\text { fundamental } \\
\text { de } 2 \text { escolas } \\
\text { municipais }\end{array}$ & $\begin{array}{l}\text { Escala Hedônica } \\
\text { facial mista de } 5 \\
\text { pontos. }\end{array}$ & $\begin{array}{l}\text { Almôndegas e } \\
\text { Nuggets } \\
\text { elaborados com } \\
\text { peixe betara } \\
\text { (Menticirrhus } \\
\text { americanos) }\end{array}$ & $\begin{array}{c}\text { Almôndegas } \\
\text { cozidas por } 5 \\
\text { minutos em } \\
\text { água. Nuggets } \\
\text { assados por } \\
15 \text { minutos. a } \\
180^{\circ} \mathrm{C} \text {. }\end{array}$ & $\begin{array}{l}\text { O índice de aceitabilidade foi } \\
\text { de } 92,4 \% \text { para o nuggets e } \\
89,4 \% \text { para as almôndegas, } \\
\text { sendo muito bem aceitos } \\
\text { pelos escolares, podendo ser } \\
\text { facilmente introduzidos na } \\
\text { alimentação escolar. }\end{array}$ \\
\hline $\begin{array}{l}\text { Veit et al. } \\
\text { (2012) }\end{array}$ & Toledo, PR & $\begin{array}{c}20 \text { alunos de } \\
8 \text { a } 10 \text { anos } \\
\text { de uma } \\
\text { escola } \\
\text { pública. }\end{array}$ & $\begin{array}{l}\text { Escala Hedônica } \\
\text { de } 5 \text { pontos. }\end{array}$ & $\begin{array}{l}\text { Bolos de } \\
\text { chocolate e de } \\
\text { cenoura com } \\
\text { peixe tilápia } \\
\text { (Oreochromis } \\
\text { niloticus). }\end{array}$ & $\begin{array}{l}\text { Assados por } \\
40 \text { minutos à } \\
180^{\circ} \mathrm{C}\end{array}$ & $\begin{array}{l}\text { O índice de aceitabilidade foi } \\
\text { de } 88,58 \% \text { para o bolo de } \\
\text { chocolate com filé de tilápia e } \\
89,99 \% \text { para o bolo de } \\
\text { cenoura com filé de tilápia. A } \\
\text { inclusão de pescado na } \\
\text { alimentação escolar se torna } \\
\text { uma alternativa promissora, } \\
\text { devendo ser incluído em } \\
\text { preparações que os alunos } \\
\text { apreciam. }\end{array}$ \\
\hline
\end{tabular}

\begin{tabular}{cccccc}
\hline Iitterer-Daltoé, & Rio Grande & Estudantes & Escala Hedônica & Peixe empanado & Assado \\
Latorres, & do Sul, RS & $\begin{array}{c}\text { de escolas } \\
\text { facial estruturada }\end{array}$ & $\begin{array}{c}\text { (Engraulis } \\
\text { anchoita) }\end{array}$ \\
Treptow, & & públicas de & de 7 pontos. & \\
Pastous- & & 5 a 18 anos & & \\
Madureira e & & $(\mathrm{n}=830)$. & & \\
Tueiroz (2013) & & & &
\end{tabular}

Observou-se uma relação inversa entre a aceitação de peixe na alimentação e a idade dos escolares possivelmente, as crianças se tornam mais críticas na sua preferência, com o aumento da idade, dificultando a inserção de um novo alimento que não faz parte das suas práticas alimentares.

\begin{tabular}{|c|c|c|c|c|c|}
\hline $\begin{array}{c}\text { Evangelista- } \\
\text { Barreto, } \\
\text { Rocha e Ledo } \\
(\mathbf{2 0 1 5})\end{array}$ & Acaraú, SE & $\begin{array}{c}60 \text { alunos de } \\
14 \text { a } 17 \text { anos } \\
\text { do Instituto } \\
\text { Federal de } \\
\text { Sergipe }\end{array}$ & $\begin{array}{c}\text { Escala Hedônica } \\
\text { de } 9 \text { pontos }\end{array}$ & $\begin{array}{l}\text { Biscoitos e sopa } \\
\text { a partir de } \\
\text { farinha de } \\
\text { tilápia } \\
\text { (Oreochromisnil } \\
\text { oticus). }\end{array}$ & $\begin{array}{c}\text { Biscoito: } \\
\text { assado a } \\
240^{\circ} \mathrm{C} ; \text { Sopa: } \\
\text { cozida }\end{array}$ \\
\hline
\end{tabular}

\begin{tabular}{|c|c|c|c|c|c|c|}
\hline $\begin{array}{l}\text { Latorres, } \\
\text { Mitterer- } \\
\text { Daltoé e } \\
\text { Queiroz (2016) }\end{array}$ & $\begin{array}{c}\text { Caçapava } \\
\text { do Sul, Rio } \\
\text { Grande do } \\
\text { Sul, RS }\end{array}$ & $\begin{array}{l}132 \text { alunos } \\
\text { de uma } \\
\text { escola } \\
\text { pública. }\end{array}$ & $\begin{array}{l}\text { Escala Hedônica } \\
\text { facial de } 7 \text { pontos } \\
\text { e Percepção } \\
\text { cognitiva }\end{array}$ & $\begin{array}{l}\text { Almôndegas de } \\
\text { peixe anchoita } \\
\text { (Engraulis } \\
\text { anchoita) no } \\
\text { molho de } \\
\text { tomate }\end{array}$ & $\begin{array}{l}\text { Cozidas no } \\
\text { molho de } \\
\text { tomate a } 150^{\circ} \\
\text { por } 15 \\
\text { minutos }\end{array}$ & $\begin{array}{l}\text { A idade foi inversamente } \\
\text { proporcional à aceitação. As } \\
\text { preferências dos mais jovens } \\
\text { foram dadas pela aparência da } \\
\text { preparação, e, à medida que } \\
\text { cresciam, suas cognições } \\
\text { tornavam-se mais abstratas. O } \\
\text { produto mostrou ser } \\
\text { potencialmente aceito na } \\
\text { alimentação escolar. }\end{array}$ \\
\hline
\end{tabular}




\begin{tabular}{|c|c|c|c|c|c|c|}
\hline $\begin{array}{c}\text { Breda, } \\
\text { Belusso, } \\
\text { Nogueira, } \\
\text { Camargo e } \\
\text { Mitterer- } \\
\text { Daltoe (2017) }\end{array}$ & $\begin{array}{c}\text { Pato } \\
\text { Branco, PR }\end{array}$ & $\begin{array}{l}142 \text { alunos } \\
(5 \text { a } 10 \\
\text { anos) de } 3 \\
\text { escolas } \\
\text { primárias. }\end{array}$ & $\begin{array}{l}\text { Escala Hedônica } \\
\text { estruturada facial } \\
\text { de } 5 \text { pontos }\end{array}$ & $\begin{array}{l}\text { Fishburguer de } \\
\text { tilápia } \\
\text { (Oreochromis } \\
\text { niloticus) }\end{array}$ & $\begin{array}{l}\text { Assados à } 180 \\
{ }^{\circ} \mathrm{C} \text { por } 40 \\
\text { minutos. }\end{array}$ & $\begin{array}{l}\text { O índice de aceitabilidade do } \\
\text { fishburguer foi de } 87 \% \text {, } \\
\text { mostrando o potencial para o } \\
\text { consumo deste alimento pelas } \\
\text { crianças. }\end{array}$ \\
\hline $\begin{array}{c}\text { Fonseca et al. } \\
\qquad(2017)\end{array}$ & $\begin{array}{c}\text { Rio Grande } \\
\text { do Sul, RS }\end{array}$ & $\begin{array}{c}25.778 \\
\text { escolares. }\end{array}$ & Escala Hedônica & $\begin{array}{l}\text { Massa, pizza, } \\
\text { polenta, batata, } \\
\text { pão e risoto com } \\
\text { molho de peixe } \\
\text { anchoita } \\
\text { (Engraulis } \\
\text { anchoita) }\end{array}$ & $\begin{array}{l}\text { Cozido/ } \\
\text { Assado }\end{array}$ & $\begin{array}{l}\text { A média de adesão foi de } \\
92,1 \% \text { para pizza, } 72,3 \% \text { para } \\
\text { polenta, } 87,5 \% \text { para batata, } \\
83,9 \% \text { para pão, } 72,9 \% \text { para } \\
\text { risoto e } 68,5 \% \text { para massa. } \\
\text { Acredita-se ser possível a } \\
\text { inclusão do pescado na } \\
\text { alimentação escolar, por meio } \\
\text { de iniciativas que promovam } \\
\text { o consumo de pescado. }\end{array}$ \\
\hline
\end{tabular}

\begin{tabular}{|c|c|c|c|c|c|c|}
\hline $\begin{array}{l}\text { Kato, Maciel, } \\
\text { Quaresma e } \\
\text { Freitas, } \\
\text { (2017) }\end{array}$ & Palmas, TO & $\begin{array}{l}120 \text { alunos } \\
\text { do ensino } \\
\text { fundamental } \\
\text { e médio }\end{array}$ & $\begin{array}{c}\text { Escala Hedônica } \\
\text { de } 5 \text { pontos. }\end{array}$ & $\begin{array}{l}\text { Arroz nutritivo, } \\
\text { salada fria de } \\
\text { macarrão e torta } \\
\text { feitos com } \\
\text { tambaqui } \\
\text { (Colossoma } \\
\text { macropomum) }\end{array}$ & $\begin{array}{l}\text { Cozido/ } \\
\text { Assado }\end{array}$ & $\begin{array}{l}\text { A salada de macarrão não foi } \\
\text { bem aceita. O arroz nutritivo } \\
\text { foi bem aceito pelo ensino } \\
\text { fundamental }(86 \%) \text { e a torta } \\
\text { foi altamente aceita, sendo o } \\
\text { fundamental } 100 \% \text { e o médio } \\
93,8 \% \text {. A aceitação de peixes } \\
\text { pelos mais jovens é maior que } \\
\text { adolescentes. Políticas que } \\
\text { promovem a ingestão de } \\
\text { peixe e um estilo de vida } \\
\text { saudável devem ser } \\
\text { estimuladas. }\end{array}$ \\
\hline
\end{tabular}

\begin{tabular}{|c|c|c|c|c|c|c|}
\hline $\begin{array}{l}\text { Neiva et al. } \\
\text { (2017) }\end{array}$ & $\begin{array}{l}\text { Itanhaém, } \\
\text { SP }\end{array}$ & $\begin{array}{c}240 \text { crianças } \\
\text { de } 9 \text { a } 12 \\
\text { anos }\end{array}$ & $\begin{array}{l}\text { Escala Hedônica } \\
\text { Facial de } 5 \\
\text { pontos }\end{array}$ & $\begin{array}{c}\text { Macarrão com } \\
\text { molho tipo a } \\
\text { Bolonhesa e } \\
\text { Escondidinho } \\
\text { de Peixe feitos } \\
\text { com pescada } \\
\text { Goete } \\
\text { (Cynoscion } \\
\text { jamaicensis) }\end{array}$ & $\begin{array}{l}\text { Cozido/ } \\
\text { Assado }\end{array}$ & $\begin{array}{l}\text { Observou-se aceitação } \\
\text { superior a } 85 \% \text { para as duas } \\
\text { preparações, sendo o } \\
\text { macarrão mais bem aceito. Há } \\
\text { viabilidade de inserção do } \\
\text { pescado na alimentação } \\
\text { escolar. }\end{array}$ \\
\hline $\begin{array}{l}\text { Lustosa-Neto } \\
\text { et al. }(2018)\end{array}$ & $\begin{array}{l}\text { Fortaleza, } \\
\text { CE }\end{array}$ & $\begin{array}{c}120 \text { crianças } \\
\text { do ensino } \\
\text { fundamental } \\
\text { do Colégio } \\
\text { Militar do } \\
\text { Corpo de } \\
\text { Bombeiros } \\
\text { do Estado } \\
\text { do Ceará }\end{array}$ & $\begin{array}{l}\text { Escala Hedônica } \\
\text { Facial Mista }\end{array}$ & $\begin{array}{l}\text { Almôndegas de } \\
\text { pirarucu } \\
\text { (Arapaima } \\
\text { gigas) e tilápia } \\
\text { (Oreochromis } \\
\text { niloticus) }\end{array}$ & Cozido & $\begin{array}{l}\text { As almôndegas de pirarucu e } \\
\text { de tilápia tiveram } 100 \% \text { de } \\
\text { aceitação podendo se } \\
\text { constituir numa grande opção } \\
\text { de aumento de consumo de } \\
\text { pescado. Além disso, } \\
\text { corrobora ao objetivo de } \\
\text { implantar um novo produto e } \\
\text { de valor agregado voltado } \\
\text { para alimentação } \\
\text { institucional. }\end{array}$ \\
\hline $\begin{array}{c}\text { Andrade } \\
\text { (2020) }\end{array}$ & $\begin{array}{l}\text { Benjamin } \\
\text { Constant, } \\
\text { AM }\end{array}$ & $\begin{array}{c}20 \\
\text { estudantes } \\
\text { de escola }\end{array}$ & $\begin{array}{l}\text { Avaliação dos } \\
\text { itens: Regular, } \\
\text { Bom e Ótimo. }\end{array}$ & $\begin{array}{l}\text { Fishburguer de } \\
\text { curimatã } \\
\text { (Prochilodus }\end{array}$ & $\begin{array}{l}\text { Submetido à } \\
\text { fritura, } \\
\text { cortado em }\end{array}$ & $\begin{array}{l}\text { O fishburger foi bem aceito } \\
\text { pelos alunos, sendo uma } \\
\text { proposta para alimentação }\end{array}$ \\
\hline
\end{tabular}




\begin{tabular}{|c|c|c|c|}
\hline $\begin{array}{l}\text { pública do } \\
\text { município. }\end{array}$ & $\begin{array}{c}\text { nigricans } \\
\text { Agassiz) }\end{array}$ & cubículo & $\begin{array}{l}\text { escolar. Foi considerado com } \\
\text { boa aparência }(60 \%) \text {, ótima } \\
\text { qualidade }(30 \%) \text {. Em relação } \\
\text { à textura, } 55 \% \text { avaliaram as } \\
\text { amostras provenientes do T2 } \\
\text { como de ótima qualidade. }\end{array}$ \\
\hline
\end{tabular}

Fonte: Autores (2021).

Cabe destacar que, no Brasil, há uma rica biodiversidade de pescado, que pode ser usada na alimentação humana, sendo os peixes mais capturados a sardinha-verdadeira, a corvina, o bonito-listrado, a pescada e a tainha, enquanto que entre os crustáceos tem-se, como principal, o camarão e, entre os moluscos bivalves, as ostras e os mexilhões (Brasil, 2011). Santos (2009), como exemplo, considera que o sururu, um molusco bivalve, pode ser usado de forma alternativa na alimentação de crianças que possuem agravos nutricionais, por sua quantidade e biodisponibilidade de ferro e zinco.

Em relação à forma de elaboração, a maioria das preparações à base de pescado não utilizava o método de fritura (Tabela 1). As frituras não devem ser ofertadas nos cardápios das escolas, uma vez que contêm uma grande quantidade de gordura, podendo prejudicar o crescimento e desenvolvimento das crianças, além de aumentar o risco de doenças como a obesidade. Além disso, é recomendado que, ao preparar a alimentação escolar, sejam adotadas formas de preparo mais saudáveis utilizando pouca quantidade de óleo, como os assados, cozidos, ensopados e grelhados (Vasconcelos et al., 2012).

Com relação aos provadores dos testes de aceitação, observou-se variação quanto à faixa etária, havendo predomínio de crianças e adolescentes. Para a maior parte dos estudos, os testes tiveram entre 20 e 240 provadores, havendo dois estudos mais amplos $(n=830$ e $n=25.778)$.

Considerando o público dos testes, cabe salientar que as crianças mais novas tendem a aceitar mais os alimentos e que, com o avançar da idade, ficam mais resistentes, sendo mais difícil de consumirem os alimentos que não façam parte dos seus hábitos alimentares (Kato, Maciel, Quaresma \& Freitas, 2017; Latorres, Mitterer-Daltoé, \& Queiroz, 2016; MittererDaltoé, Latorres, Treptow, Pastous-Madureira \& Queiroz, 2013). Além disso, muitos fatores têm sido referidos para a baixa aceitação dos peixes entre as crianças, como uma aversão ao sabor, ao cheiro e à textura, além do receio da ingestão de espinhas. Pontua-se, ainda, que a preferência familiar e a neofobia contribuem para moldar o desenvolvimento da aceitação dos peixes (Sam, Bi \& House, 2016).

O instrumento predominante para a avaliação dos testes de aceitação foi a escala hedônica, em suas variações facial (faixa etária entre 6 e 10 anos) e estruturada (faixa etária superior a 10 anos), sendo com menor número de pontos para crianças mais novas e maior número para os mais velhos. Nesse contexto, ressalta-se que, sempre que haja a introdução de um alimento novo ou quaisquer outras alterações inovadoras no cardápio da escola, é necessário realizar o teste de aceitabilidade (Resolução n. 26, 2013; Brasil, 2017).

Nas escolas públicas brasileiras, para avaliar a aceitabilidade, é preconizado o uso de escalas hedônicas específicas por faixa etária ou o teste de resto-ingestão, sendo consideradas com boa aceitação preparações que alcancem índice igual ou superior a $85 \%$ e $90 \%$, respectivamente. Caso o teste resulte em aceitação inferior a estes valores, para a reaplicação do teste de uma mesma preparação/alimento, é necessário dar um intervalo mínimo de dois meses (Brasil, 2017).

Nesse contexto, Soares et al. (2019) ressaltam a importância do monitoramento da aceitabilidade de preparações, posto que pode sinalizar a necessidade de realizar intervenções visando melhorar a aceitação dos pratos e, consequentemente reduzir o desperdício de alimentos. Dessa forma, cabe ao nutricionista responsável fazer as devidas correções sensoriais nas preparações e desenvolver atividades de educação nutricional com as crianças (Brasil, 2017). 
Quanto aos resultados de aceitabilidade dos produtos desenvolvidos, alguns estudos apresentaram índice superior a 90\%, como no caso da torta do estudo de Kato, Maciel, Quaresma e Freitas (2017), dos nuggets do estudo de Borges, Passos, Stedefeldt e Rosso (2011), da sopa do estudo de Evangelista-Barreto, Rocha e Ledo (2015), da pizza de Fonseca et al. (2017) e das almôndegas de Lustosa-Neto et al. (2018). Outros estudos obtiveram resultados de aceitabilidade próximos de 90\%, como as almôndegas de Borges, Passos, Stedefeldt e Rosso (2011), os bolos de chocolate e de cenoura com peixe de Veit et al. (2012), o fishburguer de Breda, Belusso, Nogueira, Camargo e Mitterer-Daltoe (2017) e a polenta, batata, pão e risoto com molho de peixe de Fonseca et al. (2017), o arroz nutritivo do estudo de Kato, Maciel, Quaresma e Freitas (2017) e o macarrão com molho tipo à Bolonhesa (com peixe) e escondidinho de peixe do estudo de Neiva et al. (2017). Os autores, de modo geral, consideram os produtos com bom potencial para inserção na alimentação escolar, o que favorece o alcance de modelos de alimentação saudável já estabelecidos em países desenvolvidos.

$\mathrm{Na}$ Cornualha, Inglaterra, o governo determina que as refeições escolares forneçam peixe uma vez por semana. Deste modo, no estudo de Rees, Richards e Gregory (2008) ao comparar a ingestão de alimentos e nutrientes por crianças que consumiam refeições das escolas e de crianças que utilizavam almoços embalados, foi verificado que grande parte de crianças que recebiam alimentação escolar já haviam consumido peixe. Gatenby (2007), também no Reino Unido, relata que o governo reconheceu a contribuição da alimentação escolar para a saúde geral das crianças, estipulando padrões nutricionais para escolas que fornecem almoços, devendo os peixes estar presentes em, pelo menos, um dia da semana.

No Alasca, em 2013, foi criado um programa piloto para estimular os distritos escolares a adquirir alimentos cultivados, capturados e colhidos, inclusive peixes, que possuem um elevado teor proteico (Izumi, Pickus, Contesti, Dawson \& Bersamin, 2015).

Em estudo conduzido por Donadini, Fumi e Porreta (2013), em Varese, Itália, pratos de peixe foram ofertados para pré-escolares e avaliada a aceitabilidade por meio da escala hedônica, observando-se que as crianças gostaram de dois dos pratos servidos (Filé de Solha empanado e Bacalhau com molho de tomate). Os autores ressaltam a importância do conhecimento da aceitabilidade de determinada espécie de peixe pelas crianças, para que os gestores tenham certeza de que o peixe fornecido seja completamente consumido, promovendo práticas de alimentação saudável e nutricionalmente balanceada.

Em Bizkaia, Espanha. foi conduzida uma pesquisa com 227 escolares, com idades entre seis e doze anos, para avaliar 16 pratos, incluindo quatro com pescado, sendo: pescada assada com alface, pescada à milanesa com alface, atum com pimenta e hambúrguer de cavala. Segundo os autores, as percepções hedônicas foram muito baixas, especialmente para atum com pimenta e hambúrguer de cavala. Também foi observada maior aceitabilidade do hambúrguer de cavala entre as crianças mais novas, quando comparada à de faixas etárias maiores, e que a opinião das crianças diferiu, em relação as preparações com pescado, conforme o método de cozimento (empanados ou assados), sendo preferidos os empanados (Alfaro, Rios, Arranz, \& Varela, 2020)

No Brasil, o consumo de pescado está descrito no Guia Alimentar para a População Brasileira (Brasil, 2014b), como uma das fontes proteicas, contudo, não é preconizada uma frequência de uso. Assim, considerando o potencial de oferta e a biodiversidade de pescado no país e os seus benefícios à saúde, sinaliza-se a necessidade de incentivar a utilização desses alimentos pela população, desde a infância, respeitando os diferentes aspectos culturais.

\section{Conclusão}

Em todo o mundo, diversos benefícios têm sido atribuídos à ingestão do pescado, por sua excelente qualidade proteica e lipídica e efeitos de proteção da saúde, como a redução do risco de doenças cardiovasculares e neurodegenerativas, dentre outros. Apesar disso, no Brasil, o consumo de pescado ainda é baixo, sendo que as crianças representam a faixa etária de menor consumo. 
Políticas de incentivo ao consumo de pescado e à sua inserção na alimentação escolar têm sido implementadas, todavia coexistem desafios culturais e operacionais na consecução deste objetivo. Entre os principais, observou-se a rejeição do pescado pelas crianças, um quadro que é reforçado pelas práticas alimentares e outros determinantes sociais. Além disto, mudanças recentes na conjuntura política nacional têm evidenciado o enfraquecimento destas políticas, o que afeta sobremaneira a população.

Entre os estudos que buscaram introduzir o pescado para crianças e adolescentes, observou-se que as preparações mais bem aceitas foram elaboradas a partir de peixe triturado, sem o uso de fritura. Além disso, foi possível perceber que todos os produtos tinham a carne de peixe como matéria-prima. Entre os escolares, os mais novos tenderam a aceitar mais os produtos, quando comparados aos mais velhos, o que reafirma a necessidade de trabalhos de educação alimentar e nutricional, desde a infância.

Embora a maioria dos estudos tenha sido conduzida na região Sul do país, os achados sugerem a ampliação desta proposta para outras regiões, respeitando hábitos alimentares e técnicas culinárias, de forma a manter as propriedades nutricionais e tornar as características sensoriais das preparações atrativas. Ressalta-se, que a inserção de pescado na alimentação escolar contribui para e uma alimentação mais saudável e para a saúde dos escolares, demandando a continuidade de políticas e programas que possam alcançar este objetivo.

Dados o baixo consumo de pescado entre crianças e adolescentes e os desafios para a introdução deste alimento nos cardápios escolares, sugere-se a condução de novos estudos, que possam envolver: o desenvolvimento de preparações à base de pescado que alcancem boa aceitabilidade; a associação de sistemática de educação alimentar e nutricional para este público; o respeito à cultura alimentar regional; e o potencial de aquisição do pescado local. Assume-se que conhecimentos neste campo aportam subsídios para a tomada de decisão, contribuindo para reforçar políticas públicas, tanto na área de alimentação e nutrição quanto na de desenvolvimento social.

\section{Agradecimentos}

À Fundação de Amparo à Pesquisa do Estado da Bahia (FAPESB) pela bolsa de doutorado concedida. Ao Programa de Pós-graduação em Alimentos, Nutrição e Saúde, da Universidade Federal da Bahia (UFBA), pelo apoio neste estudo.

\section{Referências}

Agência Brasil (2012). Apenas $27 \%$ das prefeituras usam pescado na merenda escolar semanalmente, diz ministro. http://memoria.ebc.com.br/agenciabrasil/noticia/2012-05-10/apenas-27-das-prefeituras-usam-pescado-na-merenda-escolar-semanalmente-diz-ministro.

Alfaro, B., Rios, Y., Arranz, S., \& Varela, P. (2020). Understanding children's healthiness and hedonic perception of school meals via structured sorting. Appetite, 144, 104466. https://doi.org/10.1016/j.appet.2019.104466.

Amaral, M. T., da Silva Aparício, G. K., de Souza, P. L., \& dos Santos, Â. M. L. (2017). Aplicação de técnologias tradicionais no beneficiamento do pescado na Região do baixo Amazonas, Estado do Pará. Revista GEINTEC-Gestão, Inovação e Tecnologias, 7(1), 3708-3721. https://doi.org/10.7198/geintec.v7i1.840.

Amorim, A. L. B. D., Rosso, V. V. D., \& Bandoni, D. H. (2016). Acquisition of family farm foods for school meals: Analysis of public procurements within rural family farming published by the cities of São Paulo state. Revista de Nutrição, 29(2), 297-306. https://doi.org/10.1590/1678-98652016000200012.

Andrade, P. F., Nakauth, A. C. S. S., Barbosa, R. R., de Lima, E. A., \& de Souza, D. L. (2020). Análise sensorial de fishburger de Curimatã (Prochilodus sp.): uma proposta para a merenda escolar. ANINC-Anuário do Instituto de Natureza e Cultura, 3(1), 142-144. https://periodicos.ufam.edu.br/index.php/ANINC/article/view/8180/5881.

Araujo, W. M. C., Montebello, N. D. P., Botelho, R. B., \& Borgo, L. A. (2008). Alquimia dos alimentos: Editora Senac Nacional.

Bombardelli, R. A., Syperreck, M. A., \& Sanches, E. A. (2005). Situação atual e perspectivas para o consumo, processamento e agregação de valor ao pescado. Arquivos de Ciências Veterinárias e Zoologia da UNIPAR, 8(2). https://www.revistas.unipar.br/index.php/veterinaria/article/view/57/38.

Borges, N. D. S., Passos, E. D. C., Stedefeldt, E., \& De Rosso, V. V. (2011). Aceitabilidade e qualidade dos produtos de pescado desenvolvidos para a alimentação escolar da Baixada Santista. Alimentos e Nutrição Araraquara, 22 (3):441-8. http://servbib.fcfar.unesp.br/seer/index.php/alimentos/article/view/1181/1149. 
Boscolo, W. R., Feiden, A., Maluf, M. L. F., \& Veit, J. C. (2009). Peixe na merenda escolar: educar e formar novos consumidores. GFM Gráfica e Editora. Brasil. (2011). Boletim estatístico da pesca e aquicultura. Ministério da Pesca e Aquicultura.

Brasil. (2014a). $1^{\circ}$ Anuário estatístico brasileiro da pesca e aquicultura: Ministério da Pesca e Aquicultura.

Brasil. (2014b). Ministério da Saúde. Departamento de Atenção Básica, Secretaria de Atenção à Saúde. Guia alimentar para a população brasileira: Ministério da Saúde.

Brasil. (2017). Ministério da Educação. Manual para aplicação dos testes de aceitabilidade no Programa Nacional de Alimentação Escolar (PNAE). Revisão e atualização CECANE UFRGS. -: Ministério da Educação.

Breda, L. S., Belusso, A. C., Nogueira, B. A., Camargo, G. H., \& Mitterer-Daltoe, M. L. (2017). Acceptance of fish hamburgers in school meals in the Southwest Region of Paraná, Brazil. Food Science and Technology, 37, 94-100. https://doi.org/10.1590/1678-457x.34016.

Domingo, J. L. (2007). Omega-3 fatty acids and the benefits of fish consumption: is all that glitters gold?. Environment International, 33(7), 993-998. https://doi.org/10.1016/j.envint.2007.05.001.

Donadini, G., Fumi, M. D., \& Porretta, S. (2013). Hedonic response to fish in preschoolers. Journal of Sensory Studies, 28(4), 282-296. https://doi.org/10.1111/joss.12043.

Evangelista-Barreto, N. S., Rocha, J. B. S., \& Ledo, C. A. S. (2015). Elaboração de biscoitos e sopa usando farinha de peixe. Arquivos de Ciência do Mar, 48 (1): 57-61. https://doi.org/10.32360/acmar.v48i1.5863.

Food and Agriculture Organization (FAO). (2016). El estado mundial de la pesca y la acuicultura. Contribución a la seguridad alimentaria y la nutrición para todos: FAO.

Food and Agriculture Organization (FAO) (2020). El estado mundial de la pesca y la acuicultura 2020. La sostenibilidad en acción: FAO.

Fonseca, S. G., Scarparo, A. L. S., Capalonga, R., de Oliveira, L. D., Madureira, L. S. P., \& da Silva, V. L. (2017). O consumo de peixe anchoita na alimentação escolar: aceitabilidade e adesão. Ciência \& Saúde, 10(4), 245-250. https://doi.org/10.15448/1983-652X.2017.4.25523.

Fundo Nacional de Desenvolvimento da Educação. (2013). Cooperação entre FNDE e MPA promove inclusão do pescado na alimentação escolar. https://www.fnde.gov.br/index.php/acesso-a-informacao/institucional/area-de-imprensa/noticias/item/4981-coopera\%C3\%A7\%C3\%A3o-entre-fnde-e-mpapromove-inclus \%C3\%A3o-do-pescado-na-alimenta\%C3\%A7\% $3 \% \mathrm{~A} 30-$ escolar.

Galvão, J. A., \& Oetterer, M. (2014). Qualidade e processamento de pescado: Elsevier.

Gatenby, L. A. (2007). Nutritional content of school meals in Hull and the East Riding of Yorkshire: a comparison of two schools. Journal of Human Nutrition and Dietetics, 20(6), 538-548. 10.1111/j.1365-277X.2007.00829.x.

Godoy, L. C. D., Franco, M. L. R. D. S., Franco, N. D. P., Silva, A. F. D., Assis, M. F. D., Souza, N. E. D., \& Visentainer, J. V. (2010). Análise sensorial de caldos e canjas elaborados com farinha de carcaças de peixe defumadas: aplicação na merenda escolar. Food Science and Technology, 30, 86-89. http://dx.doi.org/10.1590/S0101-20612010000500014.

Guimarães, S. C., Quaresma, F. R. P., Sonati, J., Zica, M. M., \& da Silva Maciel, E. (2015). Comparação do consumo de pescado em escolares e sua relação com indicadores de saúde e qualidade de vida. Revista Cereus, 7(1), 146-159. Recuperado de http://ojs.unirg.edu.br/index.php/1/article/view/825/343.

Hinnig, P. D. F., \& Bergamaschi, D. P. (2012). Itens alimentares no consumo alimentar de crianças de 7 a 10 anos. Revista Brasileira de Epidemiologia, 15(2), 324-334. https://doi.org/10.1590/S1415-790X2012000200010.

Izumi, B. T., Pickus, H., Contesti, A., Dawson, J., \& Bersamin, A. (2015). Serving fish in school meals: perceptions of school nutrition professionals in Alaska. Journal of Child Nutrition \& Management, 39(1), n1. https://schoolnutrition.org/uploadedFiles/5_News_and_Publications/4_The_Journal_ of_Child_Nutrition_and_Management/Spring_2015/860\%20JCNM\%20manuscript\%20print\%20ready.pdf.

Jabeen, F., \& Chaudhry, A. S. (2011). Chemical compositions and fatty acid profiles of three freshwater fish species. Food chemistry, 125(3), 991-996. https://doi.org/10.1016/j.foodchem.2010.09.103.

Kato, H. C. A., Maciel, E. S., Quaresma, F. R. P., Freitas, A. A. (2017). Acceptance of Dishes based on Mechanically Separated Meat of Tambaqui (Colossoma macropomum) in a Public School, Brazil. Food Science and Nutrition Technology, 2 (1): 1-8. https://www.alice.cnptia.embrapa.br/alice/bitstream/doc/1083365/1/CNPASA2017fsnt.pdf.

Komiyama, C. M., Mendes, A. A., Takahashi, S. E., Moreira, J., Borba, H. B. A., Leonel, F. R., \& Balog Neto, A. (2009). Características qualitativas de produtos elaborados com carne de frango pálida e normal. Food Science and Technology, 29(1), 38-45. https://doi.org/10.1590/S0101-20612009000100007.

Kris-Etherton, P. M., Harris, W. S., \& Appel, L. J. (2002). Fish consumption, fish oil, omega-3 fatty acids, and cardiovascular disease. Circulation, 106(21), 2747-2757. https://doi.org/10.1161/01.CIR.0000038493.65177.94.

Lange, K. W. (2020). Omega-3 fatty acids and mental health. Global Health Journal, 4(1), 18-30. https://doi.org/10.1016/j.glohj.2020.01.004.

Latorres, J. M., Mitterer-Daltoé, M. L., \& Queiroz, M. I. (2016). Hedonic and word association techniques confirm a successful way of introducing fish into public school meals. Journal of Sensory Studies, 31(3), 206-212. https://doi.org/10.1111/joss.12204.

Lima, C. F. M., Pinto, M. L., de Almeida Kato, H. C., \& Pires, C. R. F. (2017). Relato de experiência de educação nutricional para o incentivo do consumo do pescado entre escolares do ensino fundamental em Palmas, Tocantins. Revista Em Extensão, 16(1), 140-149. https://doi.org/10.14393/REE-v16n12017_rel05. 
Lustosa-Neto, A. D. (2018). Caracterização de almôndegas de pirarucu e tilápia do Nilo cultivados: aplicação na merenda escolar. Acta of Fisheries and Aquatic Resources, 6(2), 1-12. https://doi.org/10.2312/Actafish.2018.6.2.1-12.

Mazzutti, E., \& Ferreto, L. E. (2006). A desnutrição e o consumo alimentar das crianças do bairro São Francisco do Município de Salto do Lontra/PR. Varia Scientia, 6(11), 11-32. http://saber.unioeste.br/index.php/variascientia/article/view/703.

Melão, I. B. (2012). Produtos sustentáveis na alimentação escolar: o PNAE no Paraná. Caderno IPARDES-Estudos e Pesquisas, 2(2), 87-105. http://ciorganicos.com.br/wp-content/uploads/2013/09/Artigo-Final-533-2301-1-PB.pdf.

Mitterer-Daltoé, M., Latorres, J., Treptow, R., Pastous-Madureira, L., \& Queiroz, M. (2013). Acceptance of breaded fish (Engraulis anchoita) in school meals in extreme southern Brazil. Acta Alimentaria, 42(2), 275-282. http://dx.doi.org/10.1556/AAlim.42.2013.2.15.

Mohan Dey, M., Rab, M. A., Paraguas, F. J., Piumsombun, S., Bhatta, R., Ferdous Alam, M., \& Ahmed, M. (2005). Fish consumption and food security: a disaggregated analysis by types of fish and classes of consumers in selected Asian countries. Aquaculture Economics \& Management, 9(1-2), 89-111. https://doi.org/10.1080/13657300590961537.

Neiva, C. R. P., Furlan, E. F., Machado, T. M., Schattan, R. B., Costa, E. L., Tomita, R. Y. (2017). Aceitação de preparações à base de carne mecanicamente separada (CMS) de pescado na alimentação escolar. Proceedings do VII SIMCOPE. Instituto de Pesca, 62-71. https://www.pesca.sp.gov.br/simcope/proceedings/Proceedings-BIP-169.pdf.

Nekitsing, C., Hetherington, M. M., \& Blundell-Birtill, P. (2018). Developing healthy food preferences in preschool children through taste exposure, sensory learning, and nutrition education. Current Obesity Reports, 7(1), 60-67. https://doi.org/10.1007/s13679-018-0297-8.

Nota técnica $\mathrm{n}^{\circ} 004$ de 11 de julho de 2013. (2013). Dispõe sobre a inclusão de pescado na alimentação escolar. Brasília, DF. https://www.fnde.gov.br/index.php/programas/pnae/pnae-area-gestores/pnae-notas-tecnicas-pareceres-relatorios.

O'Hara, J. K., \& McClenachan, L. (2019). Factors influencing 'Sea to School'purchases of local seafood products. Marine Policy, 100, 76-82. https://doi.org/10.1016/j.marpol.2018.11.023.

Peixe BR (2019). Anuário Peixe BR da Piscicultura 2019. https://www.peixebr.com.br/Anuario2019/AnuarioPeixeBR2019.pdf.

Perez, J., Pezzuto, P. R., Andrade, H. A., Schwingel, P. R., Rodrigues-Ribeiro, M., \& Wahrlich, R. (2002). O ordenamento de uma nova pescaria direcionada ao peixe-sapo (Lophius gastrophysus) no Sudeste e Sul do Brasil. Brazilian Journal of Aquatic Science and Technology, 6(1), 65-83. https://doi.org/10.14210/bjast.v6n1.p65-83.

Pérez-Rodrigo, C., \& Aranceta, J. (2001). School-based nutrition education: lessons learned and new perspectives. Public Health Nutrition, 4(1a), 131-139. https://doi.org/10.1079/phn2000108.

Raatz, S. \& Bibus, D. (2016). Fish and fish oil in health and disease prevention: Academic Press.

Rees, G. A., Richards, C. J., \& Gregory, J. (2008). Food and nutrient intakes of primary school children: a comparison of school meals and packed lunches. Journal of Human Nutrition and Dietetics, 21(5), 420-427. https://doi.org/10.1111/j.1365-277X.2008.00885.x.

Resolução no 26 de 17 de junho de 2013. Dispõe sobre o atendimento da alimentação escolar aos alunos da educação básica no âmbito do Programa Nacional de Alimentação Escolar - PNAE. Brasília, DF. https://www.in.gov.br/materia/-/asset_publisher/Kujrw0TZC2Mb/content/id/30683767/do1-2013-06-18resolucao-n-26-de-17-de-junho-de-2013-30683763.

Ribeiro, A. L. M. S., de Oliveira, G. M., de Magalhães Ferreira, V., Pereira, M. M. D., \& de Oliveira Silva, P. P. (2009). Avaliação microbiológica da qualidade do pescado processado, importado no estado do Rio de Janeiro. Revista Brasileira de Ciência Veterinária, 16(3). http://dx.doi.org/10.4322/rbcv.2014.18.

Sam, A., Bi, X., \& House, L. (2016). Identifying the attitudes and preferences of parents and children for seafood: Summary of focus groups results. FAMRC Industry Report, 11:1-3. https://edis.ifas.ufl.edu/fe992.

Sambuichi, R. H. R., Almeida, A. F. C. S. D., Perin, G., Moura, I. F. D., \& Alves, P. S. C. (2020). Texto para Discussão (TD) $2606:$ Execução do Programa de Aquisição de Alimentos nos municípios brasileiros: Instituto de Pesquisa Econômica Aplicada Ipea.

Santos, T. M. M. (2009). Análise química e microbiológica do sururu (Mytella falcata) com e sem leite de coco. Dissertação de mestrado. Universidade Federal de Alagoas, Maceió, AL, Brasil.

Sartori, A. G. O, \& Amancio, R. D. (2012). Pescado: importância nutricional e consumo no Brasil. Segurança Alimentar e Nutricional, 19(2), 83-93. https://doi.org/10.20396/san.v19i2.8634613.

Semana do pescado. (2020). Participe da Semana do pescado. https://www.semanadopescado.com.br/participe-da-17a-semana-do-pescado.

Silva, M. K. D. (2012). Aproveitamento gastronômico de pescados oceânicos de ocorrência local em cardápios de restaurantes no litoral de Santa Catarina. Dissertação de mestrado. Universidade do Vale de Itajaí, Balneário Camboriú, SC, Brasil.

Silva, R. C., Vieira, M. M. F., Simões, J. M., \& Abdallah, P. R. (2005). A indústria pesqueira no Rio Grande do Sul-ascensão e queda. REAd-Revista Eletrônica de Administração, 11(6). https://seer.ufrgs.br/read/article/view/40619/25831.

Sinn, N., Milte, C., \& Howe, P. R. C. (2009). From ADHD to Alzheimer's: omega-3 fatty acids and mental health (pp. 611-641). AOCS press. 
Research, Society and Development, v. 10, n. 3, e2210312919, 2021

(CC BY 4.0) | ISSN 2525-3409 | DOI: http://dx.doi.org/10.33448/rsd-v10i3.12919

Soares, T. C, Soares, T. C, Barbosa, M. L., Câmara, G. B., Barbosa, M. L., de Santana Alves, I. C., \& Lima, A. R. N. (2019). Avaliação da Aceitabilidade e Perfil Nutricional da Merenda Escolar em uma Instituição Pública de Ensino na Cidade de Picos-PI. Research, Society and Development, 8(11), e138111445e138111445. http://dx.doi.org/10.33448/rsd-v8i11.1445.

Soares, P., Martinelli, S. S., Melgarejo, L., Davó-Blanes, M. C., \& Cavalli, S. B. (2015). Potencialidades e dificuldades para o abastecimento da alimentação escolar mediante a aquisição de alimentos da agricultura familiar em um município brasileiro. Ciência \& Saúde Coletiva, 20, 1891-1900. https://doi.org/10.1590/1413-81232015206.16972014.

Sociedade Nacional de Agricultura (2015). Consumo de pescado no Brasil está abaixo do recomendado pela OMS. https://www.sna.agr.br/consumo-depescado-no-brasil-esta-abaixo-do-recomendado-pela-

oms/\#: :text=Mas\%20uma\%20estimativa\%20divulgada\%20em,abaixo\%20do\%20recomendado\%20pela\%20OMS.

Tacon, A. G., \& Metian, M. (2013). Fish matters: importance of aquatic foods in human nutrition and global food supply. Reviews in Fisheries Science, 21(1), 22-38. https://doi.org/10.1080/10641262.2012.753405.

Us Food and Drug Administration \& Us Environmental Protection Agency (2017). Eating Fish: What Pregnant Women and Parents Should Know. http://www.fda.gov/Food/FoodborneIllnessContaminants/Metals/ucm393070.htm.

Vasconcelos, F. D. A. G. D., Corso, A. C. T., Caldeira, G. V., Soares, B. A. S., Machado, M. S., Kami, A. A., \& Ozcariz, S. G. I. (2012). Manual de orientação para a alimentação escolar na educação infantil, ensino fundamental, ensino médio e na educação de jovens e adultos. PNAE-CECANE.

Vasconcelos, F. D. A. G. D., Machado, M. L., Medeiros, M. A. T. D., Neves, J. A., Recine, E., \& Pasquim, E. M. (2019). Public policies of food and nutrition in Brazil: From Lula to Temer. Revista de Nutrição, 32. https://doi.org/10.1590/1678-9865201932e180161.

Veit, J. C., Freitas, M. B., Dos Reis, E. S., De Queiroz Moore, O., Finkler, J. K., Boscolo, W. R., \& Feiden, A. (2012). Desenvolvimento e caracterização de bolos de chocolate e de cenoura com filé de tilápia do nilo (Oreochromis niloticus). Brazilian Journal of Food \& Nutrition/Alimentos e Nutrição, 23(3). http://serv-bib.fcfar.unesp.br/seer/index.php/alimentos/article/view/427/1605.

Verbeke, W., Vermeir, I., \& Bruns $\varnothing$, K. (2007). Consumer evaluation of fish quality as basis for fish market segmentation. Food Quality and Preference, 18(4), 651-661. https://doi.org/10.1016/j.foodqual.2006.09.005.

Virta, A., \& Love, D. C. (2020). Assessing Fish to School Programs at 2 School Districts in Oregon. Health Behavior and Policy Review, 7(6), 557-569. https://doi.org/10.14485/HBPR.7.6.5.

Welch, A. A., Lund, E., Amiano, P., Dorronsoro, M., Brustad, M., Kumle, M., \& Slimani, N. (2002). Variability of fish consumption within the 10 European countries participating in the European Investigation into Cancer and Nutrition (EPIC) study. Public Health Nutrition, 5(6b), 1273-1285. https://doi.org/10.1079/PHN2002404.

Wine, O., Osornio-Vargas, A. R., \& Buka, I. S. (2012). Fish consumption by children in Canada: Review of evidence, challenges and future goals. Paediatrics \& Child Health, 17(5), 241-245. https://doi.org/10.1093/pch/17.5.241. 